\title{
CAPACIDAD INTELECTUAL EN MUJERES AREQUIPEÑAS SEGÚN SU ESTADO CIVIL
}

\section{Intellectual capacity in women arequipeñas according to their civil status}

Ronald Delgado Morales, Bianca López Bobbio, Luisa Mazeyra Zúñiga, Lizbeth Medina Zeballos, Sol Ojeda Cama, Rodrigo Quispe Gutiérrez, Rosmeri Taya Huamani*

Universidad Católica de Santa María

\section{Resumen}

El presente proyecto de investigación causal-comparativa se centra en el estudio de una comparación del coeficiente intelectual entre mujeres solteras y mujeres casadas de 25 años de edad en adelante en la ciudad de Arequipa. Se utilizó una muestra de 75 mujeres como mínimo teniendo 31 casadas y 46 solteras, con el fin de comparar el nivel de capacidad intelectual que presentan las mujeres Arequipeñas entre casadas y solteras de 25 años. No se encontró una relación causal entre las variables, acepando la hipótesis nula.

Palabras clave: Psicología de género, capacidad intelectual, inteligencia, estado civil, mujeres solteras y casadas.

\begin{abstract}
The present project of causal-comparative research focuses on the study of a comparison of the Intelligence Capacity between single women and married women 25 years of age and older in the city of Arequipa. We used a sample of 75 women at least having 31 married and 46 single women, in order to compare the level of intellectual capacity that women from Arequipa have between married and single 25 years old. No causal relationship between the variables was found, assuming the null hypothesis.
\end{abstract}

Keywords: Gender Psychology, intellectual capacity, intelligence, marital status, single and married women.

\footnotetext{
* Estudiantes del VII semestre de la carrera de psicología de la Universidad Católica de Santa María. Trabajo para el curso de Metodología de la Investigación. Profesor responsable: Walter Arias Gallegos. warias@ucsp.edu.pe
} 


\section{INTRODUCCIÓN}

Para la sociedad novohispana del Siglo XVIII tanto la construcción, la conservación como la reproducción eran partes transcendentales de la familia, sobre estos se localizaba la estructura de un estado colectivo. A la cabeza se encontraba el rey, el cual regía a sus hijos; es decir el hombre era quien decidía por la familia, y la mujer junto con los hijos debían acatar. Se dice que el jefe de la familia debía tener la misma benevolencia tanto con sus hijos como con su esposa (Arrom citado por Saloma, 2000).

La mujer no llegaba a decidir sobre sus bienes, todos pasaban de la administración por parte de su familia, su padre específicamente, a la del esposo cuando ella se casaba. De igual forma en el caso de una mujer que trabajaba, su sueldo se incorporaba a todos los bienes familiares.

Otro aspecto de control sobre la mujer era la conducta sexual, se la condicionaba bajo la amenaza de garantía de la transmisión de los derechos de propiedad a los hijos legítimos (Rubín citado por Saloma, 2000); mientras a un hombre no se le prohibía nada respecto a este aspecto.

Inicialmente las mujeres eran escasas en educación, sin considerar las labores domésticas, en las cuales si las preparaban. Más adelante las mujeres debían infundir principios morales, críticos y religiosos, y se les otorga la enseñanza de letras y números. Es así como la función de la mujer fue cambiando pues comenzó a alcanzar un prestigio y lugar que anteriormente no poseía, iniciando en los hogares, puesto que precedentemente los padres eran los únicos destinados a decidir cómo educaban a los hijos (Saloma, 2000).

Los políticos y los intelectuales sugerían que las mujeres deberían recibir una muy buena educación para poder cumplir todos sus roles domésticos, especialmente para la buena educación de sus hijos. Y las presentan como una unidad indisoluble.

Antiguamente, previos de los años 60, la mujer era ignorada por la sociedad, por el gobierno y hasta por la historia en sí. Sufrió maltrato, opresión y falta de derechos; siendo privada de educación, conocimiento e instrucción, como se mencionó.
El inicio del cambio, la lucha de igualdad se puede marcar notoriamente en dos siglos. En la primera etapa se logró la educación de niñas, aunque se dio de forma restringida; pocas resultaron las mujeres tratadas de la misma manera que hombres. En el siglo XX, se originaron las primeras manifestaciones por parte de mujeres por el derecho de sufragio, este fue el punto de quiebre para la historia de la mujer. Este suceso significó el logro de la igualdad de las mujeres. Desde ese momento, después de la Primera Guerra Mundial, la mujer fue adquiriendo cada vez más logros.

Pensar que, al inicio de la humanidad, en vez del patriarcado existente, existió el matriarcado. Las mujeres al dar a luz, eran consideradas las líderes de las sociedades. Pero fueron los hombres quienes poco a poco arrebataron el poder, e invirtieron la situación, oprimiéndolas.

Actualmente aún se puede evidenciar los estragos de ese pensamiento antiguo en frases o actos como: esperar a que un hombre saque a bailar a una mujer, que los hombres abran la puerta, esperar al príncipe azul, etc. Estos pensamientos pueden parecer inocentes, pero en realidad limitan a las mujeres a la dependencia de un hombre.

Estos estereotipos se presencian en la vida cotidiana, y el efecto de estos recae en las niñas. Cuando a una niña le dicen que tiene que esperar por lo que desean, en realidad la están limitando a no perseguir y trabajar duro por sus metas (Centro Universitario de estudios de la mujer [CUEG] citado por Saloma, 2000).

Pero la perspectiva de género abre el camino para insertar a la mujer en una perspectiva cultural, visibilizando y valorando el cambio en su percepción de la familia, en su integración a ella, en sus roles y en la influencia de estos cambios sobre la estructura familiar.

Especialmente en las últimas décadas este proceso ha dado a luz a diversas interpretaciones, tanto éticas, culturales como legales, sobre la pareja y la familia, así como sobre su composición e importancia. 
Como parte de formar una nueva imagen a las mujeres de este nuevo siglo, el mercado laboral está involucrado. En los últimos años la tasa de empleos y las actividades femeninas han logrado desplazar a las masculinas, debido a la igualdad ganó o está ganando, porque aún falta trabajo, un terreno importante respecto a los reclamos por parte de las mujeres de los últimos años.

Nos consta una larga lista de luchas feministas que lograron formar a la mujer de hoy, por otro lado, después de mencionar esto, se puede confirmar que aún quedan algunas interrogantes sobre la participación femenina en los países. Como un dato recogido por Jorge Paz (2013) que nos muestra que en los países que se realizó este estudio tales como son: Argentina, Bolivia, Chile y Paraguay los niveles de participación de las mujeres son muy bajos por lo cual el autor plantea una serie de hipótesis la cuales quieren dar explicación a estos niveles bajos de participación femenina como son que los países comparten aspectos geográficos, culturales y económicos, sin embargo esto no se ha comprobado científicamente.

Un poco más atrás en la historia, se encuentra a Barreda (citado por Saloma, 2000), quien expone sus ideas acerca del feminismo y del rol de una mujer en la sociedad; plateando la idea de sociedad en un carácter científico biológico, es decir, la idea de las diferencias entre los sexos fue fundamental para defender la igualdad y la libertad de las mujeres, sin embargo, estos conceptos para Barreda le resultaban vagos y sin trasfondo.

\section{Estado Civil:}

Ha cobrado relevancia las decisiones de las mujeres, se cree que debido al nuevo rol laboral otorgado, han modificado su perspectiva sobre el compromiso. Ahora una mujer soltera se dedica al cuidado de sí misma, está pendiente de sus logros, metas y objetivos planteados para su vida. Constantemente está en la búsqueda de su superación, de obtener mejores sucesos. Se centra en su profesión y lo que más le importa es sobresalir en este. Mientras que una mujer casada se dedica a hacer feliz a los miembros de la familia para así proporcionarles la mejor calidad de vida. Sin embargo, cuando una mujer decide ser madre, el cuidado de sus hijos hasta una edad determinada, debería ser su mayor prioridad. Las mujeres casadas tienen más posibilidades de ascender al ámbito ocupacional, ya que poseen el apoyo de sus parejas.

El fenómeno de la soltería mide el cambio de actitud de la mujer ante la relación de la pareja, la individuación, la autonomía personal, la libertad individual para actuar frente a la familia tradicional (Barragán, 2003). Por este motivo la variable del estado civil ha generado diversas investigaciones, las cuales han entablado características propias de las mujeres solteras. Las mujeres solteras tienen menos posibilidades de sufrir estrés, padecimientos cardiacos. Asimismo, informa que las posibilidades de deprimirse son prácticamente nulas y son personas muy independientes. Esto se debe a que tienen propósitos, metas, expectativas que desean cumplir antes de formar una familia, y se centran más en su éxito profesional.

También se ha evaluado el por qué de su estado civil, John Carney realizó una investigación en la cual explicó que las mujeres solteras son más inteligentes que las casadas puesto que ellas son más exigentes al momento de elegir a su pareja. Aunque la soltería no es de un solo tipo, al contrario, cada una de ellas experimenta situaciones distintas de acuerdo a su historia de vida (Walters, Carter, Papp y Silverstein; Barragán citado por Villareal, 2008).

En la variable del estado civil casado, se debe tomar en cuenta, según un estudio realizado por el Sistema Nacional para el Desarrollo Integral de la Familia [SNDIF] (Blázquez, 2017) , que las parejas suelen casarse a mayor edad, tienen menos hijos que antes, pero también menciona que existen más probabilidades de que nazcan hijos fuera del matrimonio.

En el caso de las mujeres casadas no hay características bien definidas, debido a la dependencia de la calidad de la relación. Las parejas presentan cualidades de personalidad y valores muy parecidos a las que se dan en el funcionamiento familiar. Para estar satisfechos con la vida debe haber una evaluación positiva del funcionamiento familiar que corresponde a las parejas emocionalmente estables, extrovertidas, 
abiertas a nuevas experiencias, amables y por último responsables.

\section{Teorías de emparejamiento}

La elección de pareja es un asunto complejo donde se consideran distintas características biológicas, sociales, emocionales y psicológicas. Visto desde un punto biológico, se tiene una cierta predisposición a sentirnos atraídos por otra persona que tenga genes compatibles a los nuestros, para así tener una descendencia fuerte y sana. Algunos estudios sugieren que el estrógeno y la testosterona influyen en el deseo sexual, especialmente en ciertas regiones del cerebro. Tanto las mujeres como los hombres buscan algunas cualidades en sus parejas, varia en el tiempo y en la cultura, pero hay algunos factores que normalmente se cumplen.

Antiguamente no se les permitía a las mujeres seleccionar a su compañero de vida, posteriormente a inicios del siglo XIX tuvo la eventualidad de optar por su cónyuge. En la actualidad se sigue explorando el por qué surge la elección de la pareja, por lo tanto, sigue siendo objeto de estudio.

Hay varios enfoques que explican el fenómeno de la búsqueda de pareja. Desde la perspectiva analítica, el hombre busca la parte femenina de sí mismo que ha perdido a fin de unirse nuevamente con ella (Fromm citado por Valdez, Gonzales y Sánchez; 2005, pág. 357). Erikson, por otra parte, menciona que se experimentan crisis de la identidad y se desea compartir la vida personal con una pareja (cfr. Stassen y Thompson citados por Valdez, Gonzales y Sánchez, 2005).

La cultura, la familia y el entorno social en que la mujer se desenvuelve predomina en el sufragio de la pareja sentimental, estos tres componentes son los mayores influyentes puesto que precisan conductas, cánones y políticas.

En el lapso de los años las culturas decretaron que el lazo de dos personas quedaba determinado como una unión erótica y financiera.

El seno familiar es indefectible para la evolución del ser humano, ya que es ahí donde adquiere diferencias significativas que le permite diferenciarse de otros miembros. En este ciclo se satisfacen las necesidades más básicas como por ejemplo la vestimenta, la comida. La existencia del individuo está determinada por la estructuración biológica como psicológica. A medida en que la persona va ascendiendo se va enfocando en las características que poseen su padre y su madre para posteriormente adecuarse parcial o cabalmente. En un futuro los hijos adoptarían la conducta de convivencia que ostentan los padres.

En un entorno social se descubre que los profesionales se enamoran de acuerdo a su posición laboral o a su conveniencia.

La teoría de la complementariedad propuesta por Winch (citado por Valdez, Gonzales y Sánchez; 1958) menciona que la elección de pareja es debida a la complementariedad, mas no por similitudes. Y para ello se necesita de dos condiciones:

a. Las mujeres buscan situaciones diferentes, pero de igual intensidad en los hombres y viceversa

b. Las necesidades son idénticas en ambos sexos, pero la intensidad varia, un ejemplo de ello sería un hombre dominante se sentirá atraído por una mujer sumisa y viceversa.

Murstein plantea la teoría estímulo-valor-rol (citado por Valdez, Gonzales y Sánchez ; 1970), que incluye tres ciclos:

- Estímulo: Es la captación corporal y tangible de la otra persona.

- Valoración: Mediante variadas charlas ambos sexos llegan a la conclusión y complementación de que se conecten de acuerdo a sus valores.

- Función: Si la persona esta idónea a actuar frente a las nupcias.

La teoría Instrumental, designada por Richard Centers (citado por Valdez, Gonzales y Sánchez; 1975), se centra en la asignación de necesidades, la cual establece que nos sentimos atraídos por personas semejantes o complementarias a nosotros.

Por otra parte Carter y Mc Goldrick propone la redefinición de la estructura, mediante la teoría del 
sistema familiar (1999), la cual permite conocerse a uno mismo y poder relacionarse con las demás personas.

Se llevó a un consenso respecto a los principales puntos de enfoque, diferenciando por sexos. En los últimos tiempos se ha descubierto que ambos sexos anhelan características particulares en la pareja, las cuales son totalmente distintas. Las mujeres establecen tipologías centrándose en el ámbito social, monetario, afectivo, la capacidad intelectual, la afinidad, la asiduidad, cavilan en la buena educación que recibirán sus hijos, así como la responsabilidad. En los 50 las mujeres valoran el prestigio, la posición social (Langhorne y Secord citados por Maté y Acarin, 2011). Corroborando esta información más adelante se muestra que las mujeres valoran más los recursos económicos y la sinceridad (Wiederman \& Allgeier citados por Maté y Acarin, 2011).

En las respuestas dadas por los universitarios estudiados, las características que se valoran para tener una pareja estable son el compromiso, la amabilidad, la sinceridad y la comprensión en general. En cambio, cuando se comparan las preferencias de hombres y mujeres se observan diferencias con respecto al atractivo físico que es más valorado por los varones, y las mujeres dan más importancia a la posición social. (Harrison \& Saced citados por Maté y Acarin, 2011, pág.43)

En contraste con los hombres que ansían una mujer con cualidades que priman en el semblante físico y corporal; según Buss et al. (citado por Maté y Acarin, 2011), la pureza y los valores que existen en ella, la personalidad ininteligible y obscura, al igual que su estado de salud y su capacidad de reproducción.

Existen algunas características tanto en hombres como en mujeres que examinan en su compañero de vida tales como, por : el físico, las emociones, la intimidad, las cualidades y personalidad, los valores, la aceptación social, el humor, el desarrollo profesional, las expectativas, la salud, las actitudes, la economía, la interacción y las creencias religiosas.

Bowen propone la teoría de la diferenciación (citado por Jankowski y Hooper, 2012), en la que los seres humanos necesitamos sentir que somos una entidad diferente que destilará a lo largo de nuestra vida, asimismo incumbimos a grupos, familias y parejas.

La diferenciación contiene tanto a la individualidad como a la conexión emocional. Se encarga de describir algunas interacciones complejas, por lo tanto, se trata de buscar la aprobación de cada uno de los miembros, la reacción que se tiene ante las expectativas, las necesidades y los trastornos. Se trata de suscitar la contribución de las familias en cuanto a los alimentos, el abrigo, así como la protección.

Existen personas con niveles altos y bajos de diferenciación, y aquellas tienen las siguientes características:

- Niveles bajos de diferenciación:

Falta de sí mismo, es por esto que tienden a formar relaciones dependientes emocionalmente, se sienten ansiosas ante la distancia, pero le tienen mucho miedo a la cercanía. Las relaciones de pareja establecidas serán ambivalentes, fusionadas y dependientes.

- Niveles altos de diferenciación:

Ellas se pueden componer por una conexión emocional y por necesidades de individualización. Consecuentemente presentan una autonomía de acuerdo a la presencia de la otra persona, influenciado por la confianza y la legitimidad. Y gracias a ello las relaciones de pareja serán dedicadas tanto al crecimiento personal como a la generosidad y no en la dependencia ni en la equidad.

Bowen propone los siguientes conceptos:

- Diferenciación del yo: Las familias piensan, sienten y actúan de una manera específica. Se encargan de reflejar los niveles de diferenciación de cada una de las personas. Se sabe que entre menos desarrollado se encuentre la diferenciación del yo en una persona el efecto será mayor en el funcionamiento. La persona deseará controlar pacífica o activamente la manera en la que el funcionamiento de los otros seres humanos se dará. Mientras que las personas con un yo poco diferenciado 
se encuentran en manos de la aceptación y aprobación de los demás. Una persona con un yo diferenciado intermedio permanece calmada y lúcida, es así como se puede distinguir entre el pensamiento de una cuidadosa evaluación de hechos y el pensamiento de la emotividad.

- Fusión: El inicio de la vida del ser humano comienza siendo dependiente, pero al pasar el tiempo el sujeto se va volviendo independiente puesto que va desarrollando cualidades y habilidades. El objetivo más importante es el de esperar que el ser humano cubra necesidades emocionales y físicas. Esto es una representación que forma parte de la construcción social que influye en el infante a través de la introspección. Las investigaciones de Ainsworth, Blehar, Warters y Wall (1978) considera que hay un vínculo entre un niño que busca de forma neurótica estar constantemente cerca de su cuidadora. Es decir que el infante estará pendiente de su madre, para que no se aleje de él ya que no tiene la seguridad de que vuelva. Esto ocurre debido a que su madre lo abandona en ocasiones y no cumple sus necesidades físicas.

- Desconexión emocional: Existe ansiedad en la relación con los seres amados. Se describe el manejo de los problemas emocionales sin resolver. Por ejemplo, cuando se evitan algunos temas delicados, aparentemente las relaciones parecen estar mejor, pero en realidad los problemas aún se encuentran ahí. Es importante aludir que las interacciones de la familia se reducen completamente si es que nos distanciamos considerablemente. Y podrían intentar relaciones íntimas para poder crear familias sustitutas en base de lo social y del trabajo. No se crea, pero son dos individuos en interacción.

Por otra parte, se han determinado tipos de elección de pareja, para un compromiso estable o una relación sin metas. En el caso de la primera, se buscan las siguientes cualidades en la persona a participar.

- La sinceridad: En el momento que una pareja se consolida, el principal componente de dicha relación debe ser la sinceridad, es el sostén de la mencionada anteriormente.

- La Inteligencia: Los malos entendidos se pueden originar en base a una mala comprensión de la información, es por eso, que la inteligencia en la pareja es esencial y primordial.

- La Empatía: El conocimiento que se tiene de la pareja, refleja el amor que se siente uno del otro, y que es importante para ambos conocer hasta el más mínimo detalle de la persona; es por eso que la empatía ayuda a que la relación mejore considerablemente.

- La amabilidad: Para la buena y agradable convivencia es necesario mostrarse atentos, desde el día en que empezaron, hasta que concluyen la relación. La actitud simpática, tierna y fraterna ayuda que la persona sea digna de ser amada y cultiva un valor predicho a lo largo de los años.

En el caso de la segunda, son aspectos más superficiales los que intervienen.

- Atractivo Físico: Es una circunstancia ineludible y hace que las personas busquen la mejor apariencia captada ocularmente, la personalidad queda en un segundo plano y poco importante.

- La simpatía: El poder brindarle a la pareja la atención necesaria, sentimientos de calidad y que se sienta lleno de emociones al estar en presencia tuya.

- La amabilidad: Cuando no hay un compromiso tan fuerte como con una relación estable, los pequeños detalles hacen la diferencia; es por eso que cuando uno de los involucrados empieza a preocuparse por el otro, el deseo y las ganas de complacer al otro aumentan.

- La personalidad: Constantemente se pesquisa una persona con la cual se pueda compartir los mismos colores, ideas, emociones para sentir que se encuentran complementados entre sí.

Todo lo anterior se define en el proceso de selección de pareja, que comienza como algo propio 
del sujeto, quien busca conectarse emocional, óptica, intelectual y moralmente. Es ahí donde influyen distintos valores, exigencias y perspectivas, que busca infundir indirecta y directamente por necesidades propias, por consiguiente, la cultura, la familia, la academia.

Debemos tener en cuenta los siguientes principios:

- No existe la pareja perfecta: Se debe ejecutar en función de valores y anti - valores, tomar en cuenta las particularidades deseadas y pensar en aquello que no nos gustaría.

- Todo ser humano es un conglomerado de cualidades y defectos: Es de suma importancia investigar cualidades necesarias que buscamos en la persona que nos acompañará en nuestra vida cotidiana.

La mayor parte de las personas nos sentimos conquistadas por otras que tienen un buen atractivo físico y corporal, así como de su personalidad. A este proceso lo llamamos química, y lo podríamos describir como una sensación especial de atracción independientemente de si es alta, baja, mediana, flaca, gorda, blanca, entre otros.

\section{INTELIGENCIA}

Actualmente con la creciente tasa de divorcios y la disminución de ceremonias de nupcias, la investigación científica ha sido orientada hacia este tema. Una de las habilidades humanas que acogió atención fue la inteligencia.

Adquiriendo la postura de Sternberg (Infra), las personas que posean dirección, adaptación y crítica poseerán relaciones estables, donde se comparta responsabilidades, y se busque ser mejor. En otras palabras, la inteligencia sería un predictor de relaciones conyugales fuertes.

Contrariamente, South (citado por Holley, Yabiku y Benin, 2006) menciona al trabajo en mujeres casadas como riesgo de divorcio. Considerando que el éxito de las personas está relacionado positivamente con la inteligencia. Esto contradice la declaración de Sternberg (citado por Holley et al., 2006). Y ser inteligente seria sinónimo de divorcio.
Basado en ambas declaraciones Holley, Yabiku \& Benin publican en 2006 un estudio de la relación entre inteligencia y divorcio. En su investigación se consideró la influencia de variables como la raza y etnia, edad de contracción de matrimonio, estado laboral, ingreso familiar, religión, y la relación de los padres. Con el control de estas variables se llegó a la asociación de estabilidad e inteligencia, debido a la identificación de objetivos de la relación, y compromiso y paciencia en cumplirlos. Además, ajustaran su comportamiento, y sus citicas sean expresadas de una manera constructiva.

Pero realizando un análisis en la definición de inteligencia, un tema controversial en sí, el ámbito de la ciencia y la investigación ha tomado más de una perspectiva y corriente. Ha mostrado relación con diversos factores, al igual que ha sido demostrado como predictor de distintas conductas.

Pioneros en acotar el término inteligencia son Benet y Simon (citados por Holley et al., 2006) quienes definen este factor como la habilidad de adaptación a diversas circunstancias. Este concepto anticuado, sin describir factores especializados, fue la base para futuras investigaciones. Continuando con el factor de adaptación aparece Hebb (citado por Pérez y Medrano, 2013), incluyendo a seres animales, contradiciendo la creencia de ese entonces, de que la inteligencia es únicamente de seres humanos. Guilford (citado por Pérez y Medrano, 2013) amplio la concepción de inteligencia al introducir 120 factores que ayudan a la comprensión de la inteligencia. Poco trabajado este concepto pierde toda objetividad al implementar tal cantidad de factores, en los cuales replanteaba los iniciales, pero cabe considerar que Guilford no poseía referencias y su intento en dividir la inteligencia para iniciar la medición es considerable.

Desarrollándose el concepto de inteligencia, se aparta de la adaptación. Berg y Sternberg (citado por Pérez y Medrano, 2013) observan la inteligencia como resolución de problemas, razonamiento o toma de decisiones. Estas fueron las primeras cualidades atribuidas al concepto, aportando así las primeras concepciones de personas inteligentes, las que dominaban estos procesos. Año seguido Perkins (citado por Sierra, Molina y Pérez, 2010) describe: 
La inteligencia está formada por tres aspectos: el poder (nivel básico de aptitud intelectual inherente a la persona; su capacidad de mejora mediante el aprendizaje es limitada), el conocimiento (dominio especifico y conocimiento general de que disponemos) y las tácticas (estrategias cognitivas o técnicas de pensamiento empleadas para usar con mayor eficacia los otros dos componentes).

Esta definición desde la perspectiva de la psicología educativa nos aporta la noción de la inclusión del término en aulas escolares. Años más tarde, Sternberg (citado por Holley et al., 2006) planteó elementos relacionados más separados, la dirección, la adaptación, y la crítica. Se regreso al elemento de adaptación, más se separa de la concepción anticuada. Se refirió a adaptación como la selección y seguimiento de la forma de acción. Por otra parte, la crítica apoya al concepto de adaptación, al referirse a una autoevaluación de la técnica empleada, para así mejorarla en la siguiente situación parecida. Gottfredson (citado por Colom y Andrés-Pueyo, 1999) al igual que Sternberg, considera característica de la inteligencia la capacidad de aprender de la experiencia; adicionando el razonamiento, planificación, resolución de problemas, pero incursiona en la consideración del pensamiento abstracto. Este factor novedoso es considerado por ver más allá de lo divisible.

A finales del siglo pasado, Gardner (citado por Pérezy Medrano, 2013) construye una definición considerada fundamental para la concepción de inteligencia, considerándola como potencial biopsicológico encargado de la actividad de procesar información. Objetando, reconocidos psicólogos desacreditan este concepto general por la orientación educativa del autor. Sin embargo, es la primera concepción completa. Encontramos, recientemente la definición de este constructo por parte de Richart (citado por Sierra et al., 2013). El autor relaciona el pensamiento bueno y productivo a la terminología de caracteres intelectuales.

Se debe, además, prestar atención a variables relacionadas a este término. Una de ellas es el desenvolvimiento laboral, que en muchos casos una carrera profesional es sinónimo de un coeficiente intelectual superior pero normalmente tiene mucha más relación con el concepto de inteligencia, pero propuesta como modo de aprendizaje la cual es adquirida de muchas formas a lo largo de nuestra vida.

Por lo cual muchas de las investigaciones que se efectuaron sobre inteligencia y trabajo llegaron a la conclusión que el producto del trabajo muchas veces es contradictorio a las afirmaciones que proporcionan el resultado de los test de inteligencia que muchas veces son aplicados en las escuelas también para poder medir el rendimiento (Gottfredson citado por Colom y Andrés-Pueyo, 1999, 1997).

Por otro lado, también se toma en cuenta las actitudes específicas como los intereses, los rasgos de la personalidad y el temperamento los cuales también son factores importantes en el mundo laboral.

Debido a la orientación femenina del trabajo, consolidaremos las características propias de las mujeres, debido a que las mujeres y los hombres son distintos, es un intento absurdo pretender que sean exactamente iguales, con las mismas capacidades, aptitudes y habilidades. Por ejemplo; el tamaño del cerebro, la distribución de este, la lateralidad, y el flujo sanguíneo, etc.

Desde el siglo antepasado se trata de comprender una cuestión suelta, si estas diferencias afectan a la inteligencia. Ellis (1894) realizó un estudio comparativo, pero solo se basó en la anatomía cerebral. Actualmente sabemos que, si bien el cerebro del hombre es más grande, no hay implicaciones intelectuales en este hecho. Y cuando incluyeron las variables de peso y altura, el punto anterior se evidenció.

Se continuó el estudio, ahora orientándose en áreas específicas del cerebro. El lóbulo frontal particularmente, debido a que es el encargado de las tareas de índole superior, encontrando a los hombres con mayor superficie parietal, y las mujeres en la parte frontal. Sin embargo, los resultados no fueron los esperados, no hay diferencias significativas. Es por eso que Cox (citado por Holley et al., 1936) proclama que el intento de percibir a las mujeres como seres inferiores debía concluir, pues no se demostraba diferencias en la inteligencia. 
MacCoby \& Jacklin (1974) afirman que las mujeres poseen mayor destreza en habilidades verbales que los hombres, la explicación de frases como "hablan hasta por los codos". Mas, acota que los hombres manejan mejor las habilidades visuo-espaciales y matemáticas. Recientemente, Colom y García-López (2002) no hallan ninguna correlación entre las variables de sexo e inteligencia.

Predominan 3 teorías sobre los estudios particulares. Estas teorías evalúan la genética, hormonas, el cerebro y el factor bio-social. Los genes permiten incrementar la velocidad de pensamiento. El segundo menciona principalmente a los estrógenos y andrógenos, al aplicarlos en momentos de adrenalina, las mujeres fueron las de mayor reacción. La teoría del cerebro hace referencia a la lateralidad, los estilos de pensamiento, etc. Y la última propuesta explica los roles sociales de ambos sexos y su inclinación a seleccionar el estilo de vida.

Aunque la interrogante permanezca confusa, el objetivo de todas estas investigaciones debe abandonar la búsqueda del sexo dominante. Este ámbito de investigación debe orientarse a la búsqueda de complementación de ambos sexos.

Con lo revisado, este trabajo, de carácter causal comparativo, se planteó comparar el nivel de coeficiente intelectual en mujeres solteras y casadas de 25 años en adelante, evaluando a ambos grupos y después realizando una comparación valorativa entre ambas.

\section{METODOLOGÍA}

\section{Muestra}

Se trabajó con una muestra no probabilística de 76 mujeres de la ciudad de Arequipa, que se ubican entre los 25 y 56 años de edad. Se optó por la recolección de datos personales como, la edad y el grado de instrucción; aparte de la variable a estudiar el estado civil. Se considero casadas a las mujeres que cuenten con un compromiso estable, optando por una relación mayor a cinco años; y se considero solteras a aquellas mujeres sin relación o con una relación menor a cinco años.

\section{Instrumento}

Se utilizó el test de Raven, el cual ofrece el factor g. El análisis factorial de la comparación de formas y el razonamiento de analogías visuales. En la prueba de Raven no se considera material aprendido en escuelas, institutos, universidades, o centros de postgrado. Esta prueba es un conjunto de 60 ítems, donde se presentan imágenes con una parte faltante, y así marcar la alternativa que continúe la serie de la imagen. Midiendo:

- Fluidez verbal: Conocida también como la conectividad y la buena articulación de las palaras como parte de unos elementos como el discurso, el volumen, el tono, la velocidad, el rimo y la pausas (Crystal y Davy 1975) como plantean estos autores se obvia la fluidez cuando se interrumpe la continuidad y la ilación de la conversaciones.

- Visión espacial: Denominada un camino más primerizo en la lingüística, por otra parte también es considerada como una actividad la cual inicio con las primeras expresiones de los seres humanos de manera gráfica, también incluye componentes como discriminación, reconocimiento, imagen, razonamiento $\mathrm{y}$ reproducción de imágenes internas y externas.

- Habilidades lógico matemáticas: Es una de las inteligencias más reconocida que se evalúan en las pruebas también es la que muchas veces ayuda en la resolución de problemas, al razonamiento y la lectura, en el cual nuestro cerebro desarrolla muchas más facciones importantes que pueden hacer que trabaje de una manera íntegra y más eficaz en otras situaciones por la práctica que emplea.

- Memoria: En un análisis muy exhaustivo acerca de nuestra memoria y su profundidad se llega a la memoria colectiva la cual posee recuerdos muy útiles en nuestro inconsciente como las tradiciones, costumbres, las reglas y la música, con esto solemos guardar puntos de referencia que muchas veces nos ayudan a recuperar la información que necesitamos cuando la requerimos, lo cual demuestra lo eficaz y eficiente de la memoria. (Halbwachs 1968) 
Con todo esto damos un panorama general de lo que es la inteligencia según diferentes autores y con la que podemos emplear esta investigación presentando así más adelante el concepto con el cual manejamos la inteligencia según lo ya estudiado.

La escala "evalúa la totalidad del desenvolvimiento intelectual". El test de matrices progresivas, dirigido a personas de 12 a 65 años, ofrece cinco rangos de inteligencia: intelectualmente superior, definidamente superior en capacidad intelectual de término medio, intelectualmente término medio, definidamente inferior en capacidad intelectual al término medio, y deficiente mental. La aplicación del test consume 40 minutos. Cuenta además con un índice de confiabilidad de 0.82 . Se aplicó mediante un formulario electrónico, aplicando solo a sujetos conocidos que cumplan con los estándares.

\section{Procedimiento}

Se procedió a solicitar la resolución del test psicológico, sin un horario determinado a los sujetos voluntarios en la ciudad de Arequipa. Se vigiló la resolución del test de Raven (RAE) de forma seria, evitando la copia entre las participantes.

\section{Resultados}

Se puede observar en la edad que las personas se encuentran entre los 20 y 56 años. También, cabe resaltar que el puntaje bruto mínimo obtenido fue de 13 puntos, mientras que el máximo de 60 puntos, que simboliza una resolución perfecta. Tanto en la asimetría y en la curtosis, todas las variables respetan el límite de 1.5; con excepción de la curtosis en el estado civil, el cual sobrepasa con un puntaje de -1.856 . Por lo tanto, los datos no poseerían una distribución normal. Se estudió a 46 mujeres de estado civil solteras y a 30 casadas (Tabla 1).

Tabla 1.

Estadísticos Descriptivos.
.Se analizó T de Student para hallar la diferencia de las varianzas entre el puntaje bruto, es decir la inteligencia, y el estado civil. La media de las solteras es de 41.48, mientras que la media de las casadas es de 39,03 . La prueba de Levene nos indica que la varianza es mayor de 0.05; por lo tanto, obtenemos que las varianzas sean diferentes. Rechazando la hipótesis nula, que simboliza las varianzas iguales. Observamos el estadístico T con su nivel de significación bilateral; en este caso es mayor que 0.05 , la conclusión es que hay compatibilidad entre la hipótesis de igualdad de medias poblacionales y las diferencias entre las medias de grupos representados; es decir de las solteras y las casadas. Las medias son iguales tanto para las solteras como para las casadas (Tabla 2).

Tabla 2 .

T de Student- Estadísticas de grupo

\begin{tabular}{lccccc}
\hline ESTADO_CIVIL & $\mathrm{N}$ & Media & $\begin{array}{c}\text { Desviación } \\
\text { estándar }\end{array}$ & $\mathrm{gl}$ & $\mathrm{P}$ \\
\hline PUNTAJE__ & & & & & \\
BRUTO Soltera & 46 & 41.46 & 12.044 & 61.77 & 0.397 \\
$\quad$ Casada & 30 & 39.03 & 12.141 & & \\
\hline
\end{tabular}

No se asumen varianzas iguales (valor de T Student: 0.853 )

\begin{tabular}{lcccccccccc}
\hline & $\mathrm{N}$ & Rango & Mínimo & Máximo & Media & Desviación estándar & Varianza & Asimetría & Curtosis \\
\hline Edad & 76 & 36 & 20 & 56 & 34.00 & 9.091 & 82.640 & .665 & -.890 \\
Grado de Instrucción & 76 & 6 & 1 & 7 & 4.13 & 1.289 & 1.662 & -.482 & -.319 \\
Estado Civil & 76 & 1 & 1 & 2 & 1.39 & .492 & .242 & .439 & -1.856 \\
Puntaje Bruto & 76 & 47 & 13 & 60 & 40.50 & 12.060 & 145.453 & -.595 & -.254 \\
Categoria & 76 & 4 & 1 & 5 & 2.80 & 1.166 & 1.361 & .345 & -.653 \\
\hline
\end{tabular}


Se incluyó el análisis de varianza o ANOVA es una colección de modelos estadísticos y sus procedimientos asociados, en el cual la varianza está particionada en ciertos componentes debidos a diferentes variables explicativas. Obtuvimos un puntaje de 0.318 de significancia, al ser menor que 0.05 rechazamos la hipótesis de igualdad de medias; por lo tanto, no existen diferencias significativas entre dichos grupos. Al observar $\mathrm{F}$ concluimos que por lo menos dos niveles del factor producen distintos efectos en el dependiente. Y se debería estudiar en qué niveles existen esas diferencias significativas. (Tabla 3 )

Tabla 3.

ANOVA

\begin{tabular}{|c|c|c|c|c|c|}
\hline \multicolumn{6}{|c|}{ PUNTAJE_BRUTO } \\
\hline & $\begin{array}{l}\text { Suma de } \\
\text { cuadrados }\end{array}$ & $\mathrm{gl}$ & $\begin{array}{l}\text { Media } \\
\text { cuadrática }\end{array}$ & F & Sig. \\
\hline Entre grupos & 1029.719 & 6 & 171.620 & 1.199 & .318 \\
\hline Dentro de grupos & 9879.281 & 69 & 143.178 & & \\
\hline Total & 10909.000 & 75 & & & \\
\hline
\end{tabular}

Correlacionando las variables de edad y puntaje bruto de la prueba de inteligencia se halló una correlación fuerte entre ambas variables, con un valor de 0.016 , siendo negativa. Podemos observar la proporción de categorías como la de deficiente mental en solteras $(f=5,10.9 \%)$ y en casadas obtenemos ( $\mathrm{f}=4,13.3 \%$ ); mientras que en la categoría de definitivamente inferior en capacidad intelectual al término medio las solteras presentan $(\mathrm{f}=15,32.8 \%) \mathrm{y}$ las casadas obtuvieron ( $\mathrm{f}=10,33.3 \%$ ); posteriormente en la categoría de intelectualmente al término medio en las solteras se observan $(f=12,26.1 \%)$ y las casadas ( $\mathrm{f}=10,33.3 \%$ ); a continuación en la categoría de definitivamente superior en capacidad intelectual al término medio en las solteras se consiguieron ( $\mathrm{f}=$ $7,15.2 \%$ ) y en las casadas ( $f=5,16.7 \%$ ); por último en la categoría intelectualmente superior las solteras adquirieron $(f=7,15.2 \%)$ y las casadas $(f=1,3.3)$. (Tabla 4).

Tabla 4

cruzada CATEGORÍAS*ESTADO_CIVIL

\begin{tabular}{|c|c|c|c|c|c|}
\hline & & & \multicolumn{2}{|c|}{ ESTADO_CIVIL } & \multirow{2}{*}{ Total } \\
\hline & & & Soltera & Casada & \\
\hline \multirow[t]{10}{*}{ CATEGORÍAS } & \multirow[t]{2}{*}{ Deficiente mental } & Recuento & 5 & 4 & 9 \\
\hline & & $\%$ dentro de ESTADO_CIVIL & $10.9 \%$ & $13.3 \%$ & $11.8 \%$ \\
\hline & \multirow{2}{*}{$\begin{array}{l}\text { Definitivamente inferior en capacidad } \\
\text { intelectual al término medio }\end{array}$} & Recuento & 15 & 10 & 25 \\
\hline & & \% dentro de ESTADO_CIVIL & $32.6 \%$ & $33.3 \%$ & $32.9 \%$ \\
\hline & \multirow[t]{2}{*}{ Intelectualmente término medio } & Recuento & 12 & 10 & 22 \\
\hline & & \% dentro de ESTADO_CIVIL & $26.1 \%$ & $33.3 \%$ & $28.9 \%$ \\
\hline & \multirow{2}{*}{$\begin{array}{l}\text { Definitivamente superior en capacidad } \\
\text { intelectual al término medio }\end{array}$} & Recuento & 7 & 5 & 12 \\
\hline & & \% dentro de ESTADO_CIVIL & $15.2 \%$ & $16.7 \%$ & $15.8 \%$ \\
\hline & \multirow[t]{2}{*}{ Intelectualmente superior } & Recuento & 7 & 1 & 8 \\
\hline & & \% dentro de ESTADO_CIVIL & $15.2 \%$ & $3.3 \%$ & $10.5 \%$ \\
\hline \multirow{2}{*}{\multicolumn{2}{|c|}{ Total }} & Recuento & 46 & 30 & 76 \\
\hline & & $\%$ dentro de ESTADO_CIVIL & $100.0 \%$ & $100.0 \%$ & $100.0 \%$ \\
\hline
\end{tabular}

$\mathrm{x}^{\wedge} 2=841.801 ; \mathrm{gl}=756 ; \mathrm{p}=0.016$ 
Los resultados de la correlación de las variables no paramétricas: estado civil y las categorías de capacidad intelectual no son significativas. (Tabla 5).

Tabla 5

Pruebas de chi-cuadrado

\begin{tabular}{lrrc}
\hline & Valor & gl & $\begin{array}{c}\text { Significación } \\
\text { asintótica } \\
\text { (bilateral) }\end{array}$ \\
\hline Chi-cuadrado de Pearson & $2,886 a$ & 4 & .577 \\
Razón de verosimilitud & 3.303 & 4 & .508 \\
Asociación lineal por lineal & 1.044 & 1 & .307 \\
N de casos válidos & 76 & & \\
\hline
\end{tabular}

\section{DISCUSIÓN}

Con el cambio de mentalidad, que actualmente se ha dado en muchas sociedades del mundo entero, ha surgido un pensamiento de la limitación que sufren las mujeres al estar acompañadas por un hombre. Este es equivoco viendo los resultados.

Como resultado de la investigación presentada es posible concluir que no existe una relación causal entre la inteligencia entre mujeres solteras y casadas en la ciudad de Arequipa. Entonces el papel de un hombre no ocupa una influencia significativa para el desarrollo de una mujer, como el caso contrario de la calidad de la relación, que sí es un determinante en su desenvolvimiento. Jhon Carney (supra), quien afirmaba que las mujeres solteras son más inteligentes que las casadas, puesto a que ellas son más exigentes al momento de elegir a su pareja, cometió un mal silogismo. En el caso de una mujer quien conoce al hombre adecuado, y logra sacar adelante la relación, se da en evidencia la inteligencia.

Observando las definiciones, se rescata a la inteligencia orientada a la vida de pareja como capacidad de adaptación a los nuevos desafíos que se presentan cotidianamente (Benet y Simon citados por Holley et. Al, 2006). Involucra la resolución de conflictos, toma de decisiones, inferencias razonables sobre el comportamiento o pensamiento del otro. Además del pensamiento abstracto (Gottfredson citado por Colom y Andres-Pueyo, 1999)
Se podría deducir que la dedicación a forjar felicidad a los miembros de la familia para así proporcionarles una mejor calidad de vida y en algunos casos la prioridad siendo madres por el cuidado de sus hijos, no tiene algún efecto notable en el desempeño intelectual de la mujer casada. Podría ser el descanso del trabajo para estar con la familia, lo que las convertiría en más inteligentes y liberales del estrés. Desde la prehistoria la familia ha sido indefectible para la evolución del ser humano. Pero siendo su desempeño muy similar a la de una mujer soltera, que según la Universidad de Padua las mujeres solteras tienden a tener propósitos, metas, expectativas que desean cumplir antes de formar una familia, y se centran más en su éxito profesional lo cual es lógico que ellas puedan tener un alto coeficiente intelectual muy favorable a comparación de las mujeres casadas. Sin embargo, en el caso de las mujeres Arequipeñas este no es el caso ya que las mujeres solteras como casadas no presentan una diferencia significativa en su coeficiente intelectual de acuerdo a este estudio. Claro está que sobre este estudio también se tiene que tomar en cuenta diversos factores ya que la gran mayoría de mujeres encuestadas tenían estudios en pregrado completo e incluso en algunos casos estudios superiores, lo cual es razonable tal desempeño en ambos grupos. Recomendamos para futuras investigaciones considerar el factor del apego, brindado por Bowen y homogeneizar los grupos de evaluación.

\section{REFERENCIAS:}

Ainsworth, M. 1). S., Blehar, M. C., Waters, E., \& Wall, 5. (1978). Patterns of attachment: A psychological study of the Strange Situation. Hillsdale, NJ: Erlbaum.

Beckman, L. (1978). The relative rewards and costs of parenthood and employment for women. Psychology of women quartely, 2(2), 215-234.

Bers, T. (1980). Perceptions of Women's Roles Among Community College Women. Oakton Community College, 4(4), 492-507.

Blázquez, E. (2017). La asistencia social en México. Una mirada desde el SNDIF. Anfora, 24 (43), 189212.

Bosh, M. (2007). Las mujeres y el liderazgo. Desde la academia,18-23. 
Carter, B. \& McGoldrick, M. (1999). Overview. In Carter, B. and McGoldrick, M. The expanded family life cycle: Individual family and social perspectives. (3rd ed). Boston MA: Allyn and Bacon.

Colom, R. y Andrés-Pueyo, A. (1999). El estudio de la inteligencia humana: recopilación ante el cambio de mileno. Psicothema, 11(3), 452-476.

Colom, R. \& García-López, O. (2002). Sex differences in fluid intelligence among high-school graduates. Personality and Individual Differences, 32, 3, 445451.

Daros, W. R. (2014). La mujer posmoderna y el machismo. Franciscanum, 5(162), 107-129.

Ellis, H. (1894). Man and woman. London: Walter Scott.

Garcia, S. (2003). Psicología y Feminismo: Una Aproximación desde la psicología social de la ciencia y epistemología feministas. Universidad Complutense de Madrid. 1-192.

German, L. (2013). La vida y la lucha de las mujeres contra la opresión. Historia de mujeres, 1-11.

Gigy, L. (1980). Self-Concept of Single Women. University of California, San Francisco, 5(2), 321340 .

Holley,P.; Yabiku,S.;Benin,M.(2006). The Relationship Between Intellingence and Divorce. Arizona State University. Tempe, 27(12), 1723-1748.

Jankowski, P.J. y Hooper, L.M. (2012). Differentiation of self: A validation study of the Bowen theory construct. Couple and Family Psychology: Research and Practice, 13, 226-243.

Levano, R.; Rozah, E.; Ferruzo, G. (2016). La Soltería en el campo misionero. Pasión latina por el mundo,1-32.

Maccoby, E. E., \& Jacklin, C. N. (1974). The psychology of sex differences. Stanford, CA: Stanford University Press.

Mate, C.; Acarin, N. (2011). Encuesta sobra la elección de pareja a estudiantes de la Universitat Pmpeu Fabra, Barcelona (20 a 27 años). Summa Psicologia Uts, 8(1), 37-46.

Fecha de recepción: 10 de octubre 2018

Fecha de aceptación: 15 de mayo 2019
Muñoz, J.; Polo, P.; Cid, V.; Buccioni, G.; Fernandez, A.; Dufey, M.; FloresPardo, L.; Beltrami, M.; Turiegano, E. (2007). Diferencias de la conducta de emparejamiento entre mujeres jóvenes universitarias de Santiago de Chile en relación con el consumo de píldoras anticonceptivas orales. Interdisciplinaria, 34(2), 351-368.

ONU Mujeres. (2016). El progreso de las mujeres en el mundo 2015-2016. Transformar las economías para realizar los derechos. Resumen. Revista Estados Feministas, 24(2), 589-614.

Paz, J. (2013). Solteras y Casadas en el mercado laboral. Una comparación entre cuatro países de américa latina. Researchgate, 1-20.

Sierra, J.; Molina, E.; Pérez, L. (2010). Pensamiento Crítico y Capacidad Intelectual. Universidad Complutense de Madrid, 15(17), 98-110.

Perez, E.; Medrano, A. (2013). Teorías contemporáneas de la inteligencia. Una revisión crítica de la literatura. Revista Latinoamérica de Ciencia Psicológica, 5(2), 105-118.

Prentice, D.; Carranza E. (2002). What women and men should be, should't be, are allowed to be, and don't have to be: the contents of prescriptive gender stereotypes. Princeton University. Nro. (26), 269-281.

Ramdas, K. (2012). Women in waiting? Singlehood, marriage, and family in Singapore. Ernesronment and plannigng.

Saloma, A. (2000). De la mujer ideal a la mujer real. Las contradicciones del estereotipo femenino en el siglo XIX. Cuicuilco, 7(18), 1-19.

Santamarina, C. (2001). La construcción de la mujer moderna en la sociedad de consumo española. Revista de Comunicación Vivat Academia, 6(28), 1-32. http://dx.doi.org/10.15178/va.2001.28.1-32

Stuven, A. (2013). La mujer de ayer y hoy: un recorrido de incorporación social y política. Instituto de Historia. 8(61), 1-22.

Valdez, J. L.; Gonzales, N. I.; Sánchez, Z. P. (2005). Elección de pareja en universitarios mexicanos. Enseñanzas e Investigación en Psicología, 10(2), 355-367. 